\title{
Chain-of-spheres calculation on the coercivities of elongated fine particles with both magnetocrystalline and shape anisotropy
}

\author{
P. C. Kuo \\ Institute of Materials Engineering, National Taiwan University, Taipei, Taiwan, Republic of China
}

(Received 11 April 1988; accepted for publication 20 July 1988)

\begin{abstract}
Using the chain-of -spheres model, coercivities of elongated $\mathrm{CrO}_{2}, \mathrm{Fe}_{3} \mathrm{O}_{4}$, and $y$ - $\mathrm{Fe}_{2} \mathrm{O}_{3}$ particles due to the combined effect of shape and crystal anisotropies are calculated. Coercivities of these particles are treated in detail with respect to the particle axis orientation. The hysteresis $100 p$ is plotted so that each point of demagnetization curve is obtained by minimizing the total energy in which shape anisotropy energy, magnetocrystalline anisotropy energy, and the interaction energy of magnetic moments with applied field are all considered. The magnetocrystalline anisotropy energy of a chain during magnetization reversal is formulated. This model is applicable to single particle or very diuted particle assembly for which the effect of particle interactions can be neglected. Calculated coercivities are compared with the published experimental data.
\end{abstract}

\section{IATRODUGTION}

Coercivity is the most important property of hard magnetic materials. In general, the coercivity of multidomain particles is lower than that of single-domain particles. Commercial magnetic recording tapes have long been made by using elongated single-donain particles dispersed in an organic matrix.

For multidomain particles, mechanisms of magnetization reversal in the particle are associated with domain-wall motion. In the case of single-domain particles, mechanisms of magnetization reversal are associated with magnetic momen rotation. On the theoretical side, several famous models are available for the calculation of the coercivity of elongated single-domain particles. Typical examples are the Stoner-Wohlarth model, ${ }^{1}$ the curling and buckling model, ${ }^{2}$ and the chain-of-spheres model. ${ }^{3}$ Usually, the comparison of theory with experiment is carried out by the separation of shape and crystal anisotropy contributions to the particle coercivity. ${ }^{4-6}$ This approach simplifies the calculation but does not meet the real situation.

The chain-of-spheres model has been widely used to study the shape anisotropy behavior of elongated fine particles. ${ }^{7-10}$ Originally Jacobs and Bean ${ }^{3}$ developed the chain-ofspheres model with the assumption that the effect of crystal anisotropy is neglected. In the present work, a modified version of the chain-of-spheres model is derived in which the mixed anisotropies of shape + crystal are considered. When the effect of crystal anisotropy is taken into account, the magnetization reversal process of the chain depends not only on the angle between the chain axis and the applied field, but also on the magnitude of the crystal anisotropy constant and the orientation of easy crystal axis with respect to the chain axis. The problem becomes complicated. The purpose of this paper is to solve this problem, which has never been tried before. Three kinds of magnetic recording particles are investigated, i.e., elongated $\mathrm{CrO}_{2}, \gamma-\mathrm{Fe}_{2} \mathrm{O}_{3}$, and $\mathrm{Fe}_{3} \mathrm{O}_{4}$ particles.
Practically, the particles in magnetic tapes are aligned or randomly oriented. As the particles in the recording fim pass by the recording head, they experience fields that vary both in magnitude and direction. Thus the angular dependence of the particle coercivity is important in this process. To study the behavior of an assembly of particles, it is necessary to understand the magnetization reversal of the individual particles. Therefore, the magnetization reversals of individual particles are investigated first. In this paper, the particles are considered as single-crystal particles, and the coercivity discussed is that of very diluted particles assembly or single particle, so the effect of particle interactions" can be neglected.

\section{TOTAL EAERGY OF ELONGATED PARTICLES DURIRG MAGNETIZATION AEVERSAL}

In the present version of the chain-of-spheres model, anisotropy of the chain is not uniaxial, but of mixed type. To describe the total energy of a chain during magnetization reversal, we suppose that the chain is fixed in a matrix of nonmagnetic material. The choice of coordinates and notations is similar to that of Jacobs and Bean. ${ }^{3}$ The chain axis and easy crystal axis are lying on the $X-Z$ plane and the applied field is directed along the $Z$ axis. When the chain is magnetically saturated along the $Z$ axis, each sphere moment of the chain is directed in this direction. We then let the applied field $\mathrm{H}$ be directed in the opposite direction and increase gradually, as shown in Fig. 1. Let $\psi$ be the angle between the chain axis and the $Z$ axis. The sphere moments $\mu$ are described by their polar angle $\theta$, with respect to the $Z$ axis, and their azimuthal angle $\phi$ measured in the $X-Y$ plane, with respect to the $X$ axis. Thus, when the magnetocrystalline anisotropy energy $W_{\text {tac }}$ is taken into account, total energy of the chain can be written as 


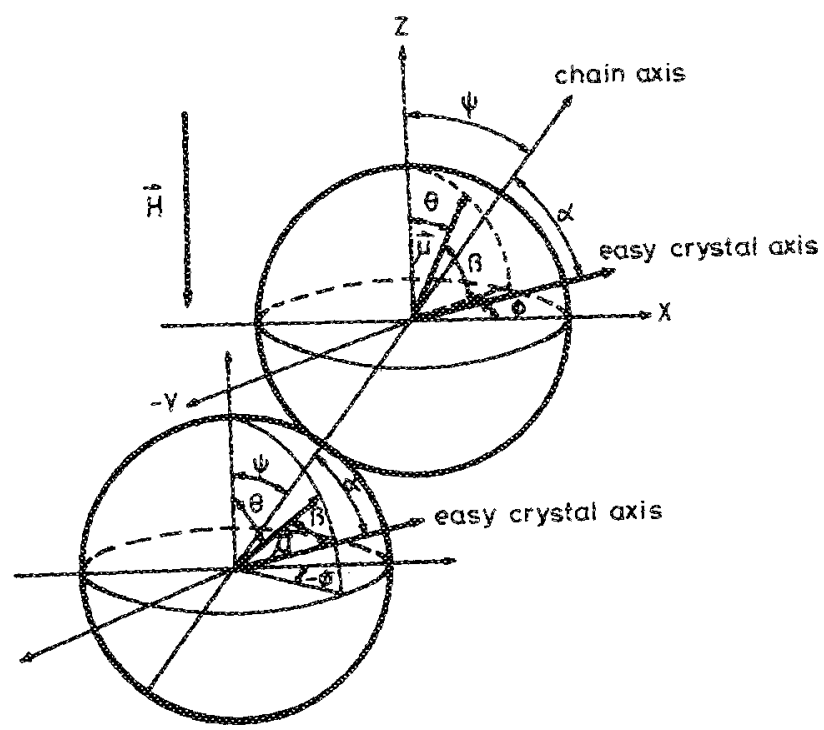

FIG. 1. Coodinate system used in the calculations.

$$
\begin{aligned}
W_{n}= & 3 n K_{n}\left(\mu^{2} / a^{2}\right)(\sin \psi \sin \theta \cos \phi \\
& +\cos \psi \cos \theta)^{2}+n L_{n}\left(\mu^{2} / a^{3}\right) \\
& \times\left(\sin ^{2} \theta \cos 2 \phi+\cos ^{2} \theta\right) \\
& +n M_{n}\left(\mu^{2} / a^{3}\right)+W_{\mathrm{rnc}}+n \mu H \cos \theta
\end{aligned}
$$

where $n$ is the axial ratio (length/width) of the chain and $a$ is the sphere diameter. $K_{n}, L_{n}$, and $M_{n}$ are parameters dependenê on $n .^{3}$

\section{A. Magnetocrystallne anisotropy energy of dongated $\mathrm{CrO}_{2}$ particles}

$\mathrm{CrO}_{2}$ has a tetragonal crystal structure with cell parameters of $a=4.423 \AA$ and $c=2.917 \AA .{ }^{12}$ The reedie axis of elongated $\mathrm{CrO}_{2}$ particles is the $c$ axis ${ }^{13}$ According to Rodbell et $a l,,^{14,15}$ the magnetocrystalline easy axis is also along the $c$ axis.

The magnetocrystalline anisotropy energy of a $\mathrm{CrO}_{2}$ chain can be written as

$$
W_{\mathrm{mc}}=n(4 / 3) \pi(a / 2)^{3} K_{1} \sin ^{2} \beta
$$

where $K_{1}$ is the first-order magnetocrystalline anisotropy constant and $\beta$ is the angle between sphere moment $\mu$ and easy crystal axis. Let $r$ be the unit vector of easy crystal axis. Then $\alpha=0^{\circ}$ for the elongated $\mathrm{CrO}_{2}$ particle and

$$
r=r \sin \psi \hat{x}+r \cos \psi \hat{z} \text {. }
$$

To the odd sphere we have

$$
\mu=\mu \sin \theta \cos \phi \hat{x}+\mu \sin \theta \sin \psi \hat{y}+\mu \cos \theta \hat{z} .
$$

To the even sphere we obtain

$$
\mu=\mu \sin \theta \cos \phi \hat{x}-\mu \sin \theta \sin \phi \hat{y}+\mu \cos \theta \hat{z} .
$$

Therefore,

$$
\begin{aligned}
\beta & =\cos ^{-1}(r \cdot \mu / r \mu) \\
& =\cos ^{-1}(\sin \theta \cos \phi \sin \psi+\cos \theta \cos \psi),
\end{aligned}
$$

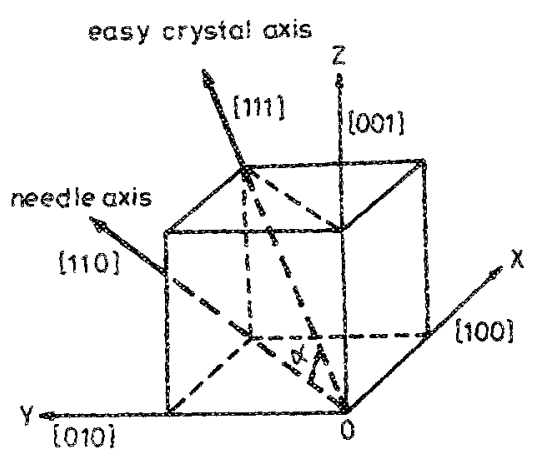

FIG. 2. Structure of elongated $\mathrm{Fe}_{3} \mathrm{O}_{4}$ and $\gamma-\mathrm{Fe}_{2} \mathrm{O}_{3}$ particles.

$$
W_{\mathrm{me}}=\frac{1}{6} \pi a^{3} n K_{\mathrm{l}} \mathcal{A}(\theta, \phi, \psi),
$$

where

$$
\begin{aligned}
A(\theta, \phi, \psi)= & \sin ^{2} \beta \\
= & 1-\sin ^{2} \theta \cos ^{2} \phi \sin ^{2} \psi \\
& -\frac{1}{2} \sin 2 \theta \cos \phi \sin 2 \psi \\
& -\cos ^{2} \theta \cos ^{2} \psi
\end{aligned}
$$

\section{Magnetocrystalline anisotropy energy of elongated $\mathrm{Fe}_{3} \mathrm{O}_{4}$ and $\gamma-\mathrm{Fe}_{2} \mathrm{O}_{3}$ particies}

Elongated $\mathrm{Fe}_{3} \mathrm{O}_{4}$ and $\gamma-\mathrm{Fe}_{2} \mathrm{O}_{3}$ particles are usually synthesized by the conversion of acicular FeOOH particles. ${ }^{16}$ $\mathrm{Fe}_{3} \mathrm{O}_{4}$ has an inverse cubic spinel structure with cell parameter of $a=8.39 \AA . \gamma-\mathrm{Fe}_{2} \mathrm{O}_{3}$ also crystallizes in an inverse spinel strueture with a cubic unit cell and $a=8.33 \AA$. The easy magnetocrystalline axis of $\mathrm{Fe}_{3} \mathrm{O}_{4}$ and $\gamma-\mathrm{Fe}_{2} \mathrm{O}_{3}$ is [111], ${ }^{17,18}$ and needle axis of these two elongated particles is $[110],{ }^{19,20}$ as shown in Fig. 2.

For the cubic crystal chain of $\mathrm{Fe}_{3} \mathrm{O}_{4}$ and $\gamma-\mathrm{Fe}_{2} \mathrm{O}_{3}$, let the magnetic moment $\mu$ make angles $\beta_{1}, \beta_{2}, \beta_{3}$ with the crystal axes [100], [010], and [001], respectively, and let $\alpha_{1}, \alpha_{2}$, and $\alpha_{3}$ be the cosine of these angles. Then the crystal anisotropy energy of a chain can be written as

$$
\begin{aligned}
W_{\mathrm{rac}}= & \frac{4}{3} \pi\left(\frac{a}{2}\right)^{3} K_{1}\left[n+\left(\frac{1-(-1)^{n}}{2}\right) / 2\right. \\
& \times\left(\alpha_{1}^{2} \alpha_{2}^{2}+\alpha_{2}^{2} \alpha_{3}^{2}+\alpha_{3}^{2} \alpha_{1}^{2}\right) \\
& \left.+n-\left(\frac{1-(-1)^{n}}{2}\right) / 2\left(\alpha_{1}^{2} \alpha_{2}^{2}+\alpha_{2}^{2} \alpha_{3}^{2}+\alpha_{3}^{2} \alpha_{1}^{2}\right)\right]
\end{aligned}
$$

In this equation, the first term is the crystal anisotropy energy of odd spheres and the second term is the crystal anisotropy energy of even spheres in the chain.

Let $r_{1009} r_{010}$, and $r_{001}$, be the unite vectors of [100], [010], and [001], respectively. Then the projections of $\mathbf{r}_{100}$ $x_{010}$, and $r_{001}$ on the chain axis will be $r_{100} \cos 45^{\circ}$, $r_{010} \cos 45^{\circ}$, and $r_{001} \cos 90^{\circ}$, respectively. The vectors $r_{100 \%}$ 
$r_{010}$, and $x_{001}$ in the coordinate system of Fig. 1 can be expressed as

$$
\begin{aligned}
r_{100}= & r_{100} \cos 45^{\circ} \cos \left(90^{\circ}-\psi\right) \hat{x}+r_{100} \cos 45^{\circ} \cos 90^{\circ} \hat{y} \\
& +r_{100} \cos 45^{\circ} \cos \psi \hat{z} \\
= & -\frac{\sqrt{2}}{2} r_{100} \sin \psi \hat{x}+\frac{\sqrt{2}}{2} r_{100} \cos \psi \hat{z} \\
r_{010}= & \frac{\sqrt{2}}{2} r_{010} \sin \psi \hat{x}+\frac{\sqrt{2}}{2} r_{010} \cos \psi \hat{z} \\
r_{001}= & r_{00} \hat{y} .
\end{aligned}
$$

Thus, for the odd spheres we have

$$
\begin{aligned}
\alpha_{1} & =r_{100^{\circ}} \mu^{\prime} / r_{100} \mu \\
& =\frac{\sqrt{2}}{2} \sin \theta \cos \phi \sin \psi+\frac{\sqrt{2}}{2} \cos \theta \cos \psi .
\end{aligned}
$$

$$
\begin{aligned}
& \alpha_{2}=\frac{\sqrt{2}}{2} \sin \theta \cos \phi \sin \psi+\frac{\sqrt{2}}{2} \cos \theta \cos \psi, \\
& \alpha_{3}=\sin \theta \sin \phi .
\end{aligned}
$$

For the even spheres we obtain

$$
\begin{aligned}
& \alpha_{1}=\frac{\sqrt{2}}{2} \sin \theta \cos \phi \sin \psi+\frac{\sqrt{2}}{2} \cos \theta \cos \psi, \\
& \alpha_{2}=\frac{\sqrt{2}}{2} \sin \theta \cos \phi \sin \psi+\frac{\sqrt{2}}{2} \cos \theta \cos \psi, \\
& \alpha_{1}=-\sin \theta \sin \phi .
\end{aligned}
$$

Substituting Eqs. (11) and (12) into Eq. (9), we get

$$
W_{\mathrm{ine}}=n \frac{1}{6} \pi a^{3} K_{1} A(\theta, \phi, \psi),
$$

where

$$
\begin{aligned}
A(\theta, \phi, \psi)= & \frac{1}{4} \sin ^{4} \theta \sin ^{4} \psi \cos ^{4} \phi+\sin ^{3} \theta \cos \theta \sin ^{3} \psi \cos \psi \cos ^{3} \phi+\frac{3}{2} \sin ^{2} \theta \cos ^{2} \theta \sin ^{2} \psi \cos ^{2} \psi \cos ^{2} \phi \\
& +\sin \theta \cos ^{3} \theta \sin \psi \cos ^{3} \psi \cos \phi+\sin ^{4} \theta \sin ^{2} \psi \cos ^{2} \phi \sin ^{2} \phi+2 \sin ^{3} \theta \cos \theta \sin \psi \cos \psi \cos \phi \sin ^{2} \phi \\
& +\cos ^{4} \theta \sin ^{2} \theta \cos ^{2} \psi \sin ^{2} \phi+\frac{1}{4} \cos ^{4} \theta \cos ^{4} \psi
\end{aligned}
$$

According to the chain-of-spheres model, there are two magnetization reversal mechanisms, i.e., chain-of-spheres parallel rotation mechanism and fanning mechanism. In the following, two theoretical situations are of interest: that of perfectly aligned chains and that of randomiy oriented chains.

\section{\%. COEACIVITY OF ALGGNED PAPTICLE $\left(\psi=0^{\circ}\right)$}

\section{A. Parallel rotation mechanism}

If the chain is parallel to the applied field, we first examine the case of parallel rotation mechanism, i.e., $\phi=0$ or $\phi=\pi$.

\section{8. $\mathrm{CrO}_{2}$ particle}

In this case, total energy of elongated $\mathrm{CrO}_{2}$ particles during magnetization reversal from Eqs. (7), (8), and (1) will be

$$
\begin{aligned}
W_{n}= & \left(\mu^{2} / a^{3}\right) n K_{n}\left(1-3 \cos ^{2} \theta\right)+\frac{1}{6} n \pi a^{3} K_{1}\left(1-\cos ^{2} \theta\right) \\
& +n \mu H \cos \theta .
\end{aligned}
$$

During magnetization reversal, the sphere moment is diffcult to be reversed by the applied field owing to magnetic anisotropy forces. When the reverse field increases gradually, the state-of-sphere moment just changes from stable to unstable state at some critical angles. Coercivity of the chain is equivalent to the critical applied field which just overcomes these anisotropy forces. To calculate the coercivity of the chain, the equations to be solved simultaneously are

$$
\frac{\partial W_{n}}{\partial \theta}=0
$$

and

$$
\frac{\partial^{2} W_{n}}{\partial \theta^{2}}=0
$$

Since the magnetization of the particle is

$$
M_{s}=\frac{6}{\pi} \frac{\mu}{a^{3}},
$$

the critical angles of $\mathrm{CrO}_{2}$ obtained from Eqs. (15), (16), and (17) are

$$
\theta_{\mathrm{c}}=0, \pi
$$

and the critical applied field is

$$
H_{c}=\pi M_{s} K_{n} \cos \theta_{c}+\left(2 K_{1} / M_{s}\right) \cos \theta_{c} \text {. }
$$

Since this single particle coercivity applies to each particle of the well-aligned particles assembly, it also represents the coercivity of the assembly.

\section{2. $\mathrm{Fe}_{3} \mathrm{O}_{4}$ and $\mathrm{\gamma}+\mathrm{Fe}_{2} \mathrm{O}_{3}$ particles}

From Eqs. (13), (14), and (1), the total energy of elongated $\mathrm{Fe}_{3} \mathrm{O}_{4}$ or $\gamma-\mathrm{Fe}_{2} \mathrm{O}_{3}$ particles during parallel rotation reversal is

$$
\begin{aligned}
W_{n}= & \left(\mu^{2} / a^{3}\right) n K_{n}\left(1-3 \cos ^{2} \theta\right) \\
& +\frac{1}{24} \pi a^{3} n K_{1} \cos ^{4} \theta+n \mu \cos \theta
\end{aligned}
$$

The critical angles obtained from Eqs. (20), (16), (17), and (18) are

$$
\theta_{\mathrm{c}}=0, \pi \text { and } \theta_{\mathrm{c}}=\cos ^{-1}\left( \pm \sqrt{\pi M^{2} K_{n} / 3 K_{1}}\right)
$$
and the critical applied field is

$$
H_{\mathrm{c}}=\pi M_{\mathrm{s}} X_{n} \cos \theta_{\mathrm{c}}-\frac{K_{1}}{M_{s}} \cos ^{3} \theta_{\mathrm{c}}
$$

\section{Fanning mechanism}

\section{$1.6 \mathrm{CrO}_{2}$ particle}

If the magnetization reversal mechanism is symmetric fanning mechanism, i.e., $\phi=(\pi / 2)$, total energy of elongated $\mathrm{CrO}_{2}$ particles from Eqs. (7), (8), and (1) will be

P. C. Kuo 5073 

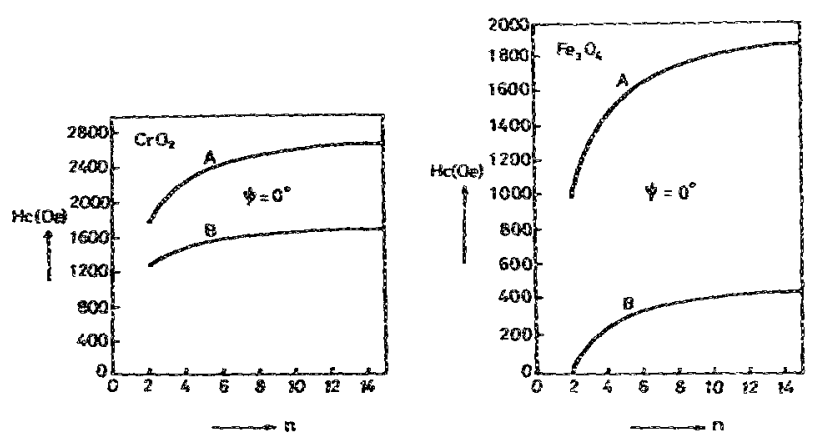

$$
\begin{aligned}
W_{n}= & -3 n K_{n}\left(\mu^{2} / a^{3}\right) \cos ^{2} \theta+n L_{n}\left(\mu^{2} / a^{3}\right) \cos 2 \theta \\
& +n M_{n}\left(\mu^{2} / a^{3}\right)+\frac{1}{b} \pi a^{3} n K_{3}\left(1-\cos ^{2} \theta\right) \\
& +n \mu H \cos \theta .
\end{aligned}
$$

Substituting Eq. (22) into Eqs. (16) and (17), and using Eq. (18), we can get the critical angles

$$
\theta_{c}=0, \pi,
$$

and the critical applied field

$$
\begin{aligned}
H_{\mathrm{c}}= & \pi M_{s} K_{n} \cos \theta_{c}-(2 / 3) \pi M_{s} L_{n} \\
& \times \cos \theta_{c}+\left(2 K_{1} / M_{s}\right) \cos \theta_{c} .
\end{aligned}
$$

\section{2. $\mathrm{Fe}_{2} \mathrm{O}_{4}$ and $\gamma-\mathrm{Fe}_{2} \mathrm{O}_{3}$ particles}

From Eqs. (13), (14), and (1), total energy of the elongated $\mathrm{Fe}_{3} \mathrm{O}_{4}$ or $\gamma-\mathrm{Fe}_{2} \mathrm{O}_{3}$ particles during fanning reversal will be

$$
\begin{aligned}
W_{n}= & -3 n K_{n}\left(\mu^{2} / a^{3}\right) \cos ^{2} \theta+n L_{n}\left(\mu^{2} / a^{3}\right) \cos 2 \theta \\
& +n M_{n}\left(\mu^{2} / a^{3}\right)+\frac{1}{24} \pi a^{3} n K_{1} \\
& \times\left(\cos ^{4} \theta+4 \cos ^{4} \theta \sin ^{2} \theta\right)+n \mu H \cos \theta .
\end{aligned}
$$

Substituting Eq. (24) into Eqs. (16) and (17) and using Eq. (18), we have the critical angles

$\theta_{c}=0, \pi$

$\theta_{c}=\cos ^{-1}\left[ \pm\left(1 \pm \sqrt{\frac{1-\frac{8 \pi M_{s}^{2}}{15 K_{1}}\left(K_{n}-\frac{2}{3} L_{n}\right)}{4}}\right)^{1 / 2}\right]$

and the critical applied field

$$
\begin{aligned}
H_{c}= & \pi M_{s} K_{n} \cos \theta_{c}-\frac{2}{3} \pi M_{s} L_{n} \cos \theta_{c} \\
& -\left(K_{l} / M_{s}\right)\left(\cos ^{3} \theta_{c}-2 \cos ^{5} \theta_{c}\right. \\
& \left.+4 \cos ^{3} \theta_{c} \sin ^{2} \theta_{c}\right) .
\end{aligned}
$$

At room temperature, the vaiues of $M_{s}$ and $K_{1}$ for $\mathrm{CrO}_{2}$ are $483 \mathrm{G}$ and $2.5 \times 10^{5} \mathrm{erg} / \mathrm{cm}^{3}$, respectively, ${ }^{16}$ for $\mathrm{Fe}_{3} \mathrm{O}_{4}$

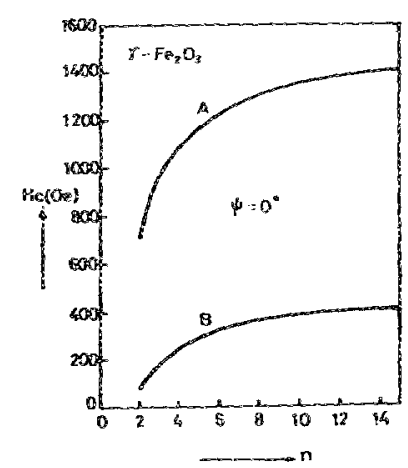

FIG. 3. Variations of coercivity with chain length (axial ratio) of $\mathrm{CrO}_{2}$, $\mathrm{Fe}_{3} \mathrm{O}_{4}$, and $\gamma-\mathrm{Fe}_{2} \mathrm{O}_{3}$ particles that are parallei to the applied field. Curve $A$ is calculated from the chain-of-spheres parallel rotation mechanism. Curve $B$ is calculated from the symmetric fanning mechanism.

are $483 \mathrm{G}$ and $2.5 \times 10^{5} \mathrm{erg} / \mathrm{cm}^{3}$, respectively, ${ }^{16}$ for $\mathrm{Fe}_{3} \mathrm{O}_{4}$ are $480 \mathrm{G}$ and $-1.1 \times 10^{5} \mathrm{erg} / \mathrm{cm}^{3,21}$ and for $\gamma-\mathrm{Fe}_{2} \mathrm{O}_{3}$ are $375 \mathrm{G}$ and $-4.64 \times 10^{4} \mathrm{erg} / \mathrm{cm}^{3}$. ${ }^{16}$ Figure 3 shows the coercivities versus $n$ of elongated $\mathrm{CrO}_{2}, \mathrm{Fe}_{3} \mathrm{O}_{4}$, and $\gamma-\mathrm{Fe}_{2} \mathrm{O}_{3}$ particles. These coercivities are obtained from Eqs. (19), (21), (23), and (25) by inspection. Curves A and B are calculated from the chain-of-spheres parallel rotation mechanism and symmetric fanning mechanism, respectively. It is found that the coercivity of $\mathrm{CrO}_{2}$ calculated from fanning mechanism is in good agreement with the largest value observed by Knowles, ${ }^{22} 1260$ Oe. The remanent coercivities of single $\gamma-\mathrm{Fe}_{2} \mathrm{O}_{3}$ particles measured by $\mathrm{K}_{2}$ nowles ${ }^{23}$ are in the range of 300 and 10500 . These values are also inconsistent with the theoretical values of Fig. 3. We conjecture that for the $\gamma-\mathrm{Fe}_{2} \mathrm{O}_{3}$ particles whose coercivities are higher than $800 \mathrm{Oe}$ may be reversed by the chain-of-spheres paraliel rotation mechanism and the particles whose coercivities are lower than 800 Oe may be reversed by the fanning mechanism.

\section{ANGULAR DEPENDENCE OF THE COERCIVITY}

If the chain is not paraliel to the applied field, to determine the hysteresis loop of a chain making an angle $\psi$ with the applicd field, we must calculate the equilibrium value of $\theta$ and $\phi$ at various values of the reverse field $H$. The equations to be solved simultaneously are

$$
\left(\frac{\partial W_{n}}{\partial \theta}\right)_{\phi}=0
$$

and

$$
\left(\frac{\partial W_{n}}{\partial \phi}\right)_{\theta}=0
$$

\section{A. $\mathrm{CrO}_{2}$ particle}

From Egs. (1), (7), (8), and (26), we have the relation between $H$ and $\theta$ for the elongated $\mathrm{CrO}_{2}$ particle:

$H \sin \theta=-\pi M_{s} K_{n}\left(\sin \theta \cos \theta \sin ^{2} \psi \cos ^{2} \phi+\cos ^{2} \theta \sin \psi \cos \psi \cos \phi-\sin ^{2} \theta \sin \psi \cos \psi \cos \phi-\sin \theta \cos \theta \cos ^{2} \psi\right)$

$$
+\frac{1}{3} \pi M_{5} L_{n} \sin \theta \cos \theta(\cos 2 \phi-1)+\frac{K_{1}}{M_{s}}\left(\frac{\partial A}{\partial \theta}\right)_{\phi} .
$$



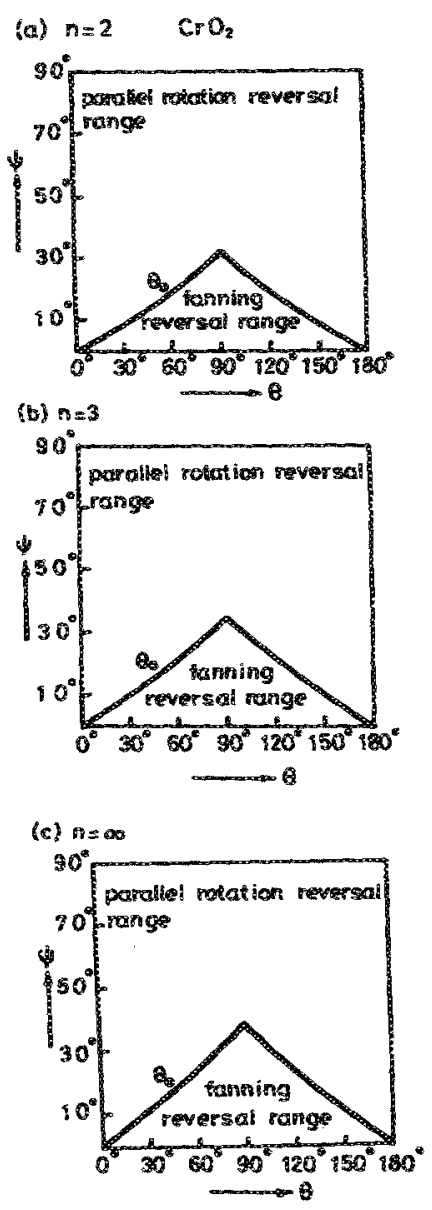

And the solution of Eq. (27) is

$$
\sin \phi=0, \phi=0, \pi,
$$

or

$$
\begin{aligned}
& \frac{1}{4} \pi M_{s} K_{n}\left(4 \cos \phi \sin ^{2} \psi \sin ^{2} \theta+\sin 2 \psi \sin 2 \theta\right) \\
& \quad-\frac{2}{3} \pi M_{s} L_{n} \sin ^{2} \theta \cos \phi+\left(K_{1} / 2 M_{s}\right) \\
& \quad \times\left(4 \sin ^{2} \theta \sin ^{2} \psi \cos \phi+\sin 2 \theta \sin 2 \psi\right)=0 .
\end{aligned}
$$

Equation (29) indicates parallel rotation mechanism and Eq. (30) is some sort of fanning mechanism.

To a given $\psi$, the $\theta$ ranges of parallel rotation and fanning mechanisms can be obtained from the necessary condition of stable state, i.e.,

$$
\left(\frac{\partial^{2} W_{n}}{\partial \phi^{2}}\right)_{\theta}>0
$$

For elongated $\mathrm{CrO}_{2}$ particles, the $\theta$ ranges of the parallel rotation mechanism and the fanning mechanism during the reversal at various $\psi$ are shown in Fig. 4 . When $\psi$ is larger than the upper bound angle $\psi_{03}$ the whole reversal process is a parallel rotation mechanism. The angle $\psi_{0}$ increases as $n$ is increased. For $n=2, n=3$, and $n=\infty$, the $\psi_{0}$ angles are $32.5^{\circ}, 34.5^{\circ}$, and $38^{\circ}$, respectively. If $\psi$ is smaller than the angle $\psi_{0}$, there are two reversal mechanisms included in the reversal process, i.e., parallel rotation mechanism and fanning mechanism. The $\theta$ range of fanning mechanism decreases as $\psi$ is increased. To the fanning mechanism, Eq. (30) may have several different solutions for the given $\psi$ and Q. Within these solutions, the physically possible state is the state that has the smallest angle $\theta$. The hysteresis loop can be synthesized by plotting the relative resolved magnetization $M / M_{s}=\cos \theta$ vs $H$. According to the numerical calculations, the representative hysteresis loop at various angle $\psi$ for $n=2$ and $n=\infty$ are shown in Fig. 5. For $\psi<\psi$, the angle $\theta$ is increased gradually as $H$ increases, there is a discontinuous change just at the angle $\theta_{0}$ marking a transformation from parallel to fanning reversal, as shown in Fig. 4. Since the applied field needed to permit the reversals in fanning reversal range are lower than those of parallel rotation range, the coercivity is determined by the transformation angle $\theta_{0}$. For $\psi \geqslant \psi_{0}$, the coercivity can be derived from Eqs. (28) and (29) by taking $\theta=90^{\circ}$.

To the parallel rotation mechanism, the whole reversal process is described by $\phi=0$ and $\phi=\pi$ for any $\psi$. Figure 6(a) shows the variation of coercivities with angle $\psi$. These coercivities are calculated from the combined effect of shape and crystal anisotropy. Solid curves are calculated from the parallel rotation mechanism and dotted curves are calculated from the fanning mechanism. Figure $6(b)$ shows the

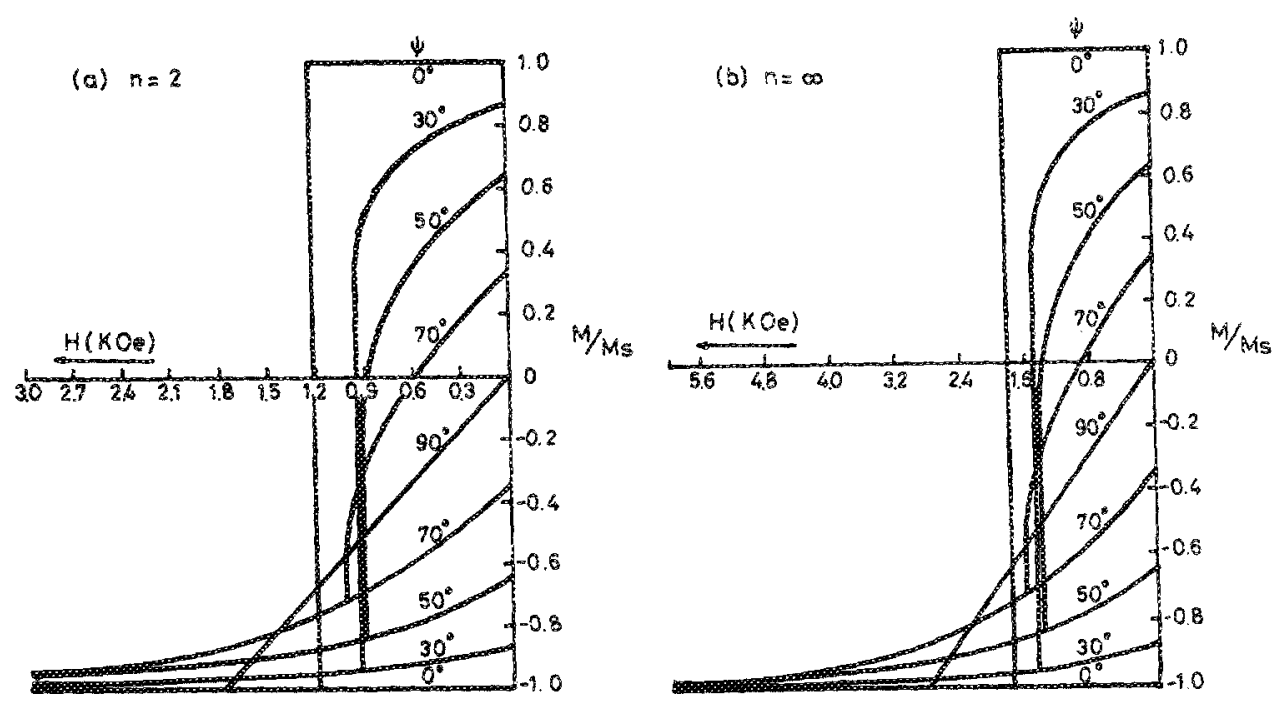

FIG. 5. Hysteresis loops of the farning mechanism for the $\mathrm{CrO}_{2}$ particle which orierted at various angles $\psi$ to the applied field. Particle axial ratio: (a) $n=2$; (b) $n=\infty$ 


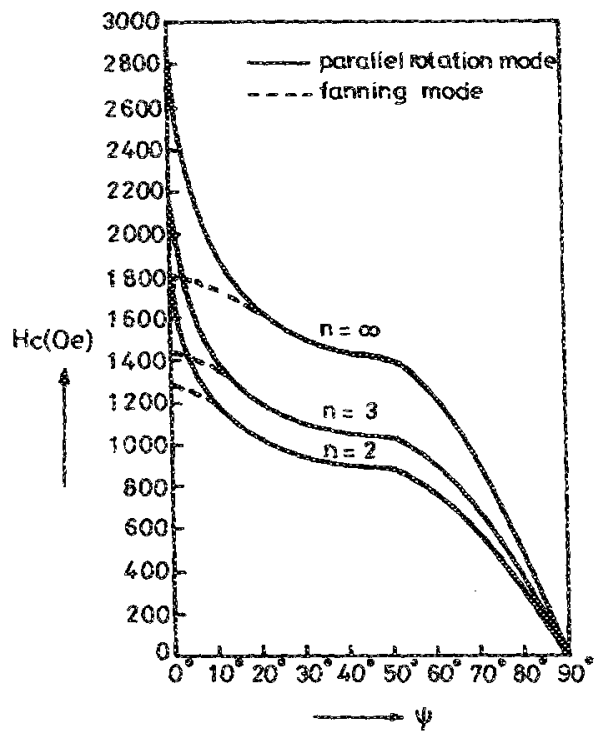

(a)

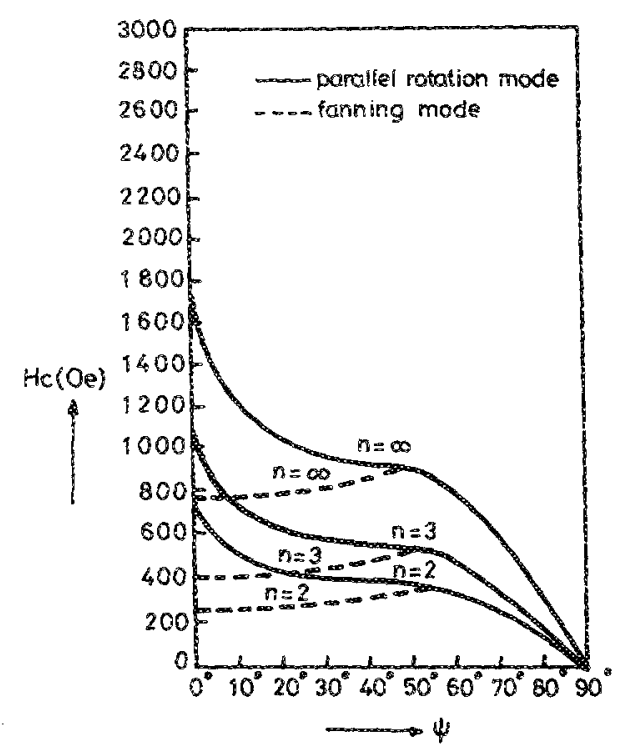

(b)
FIG. 6. Angular variation of the coercivity for $\mathrm{CrO}_{2}$ particles with various axial ratio. (a) Curves with mixed anisotropies of shape and crystal. (b) Curves with shape anisotropy. shape controlled coercivities of elongated $\mathrm{CrO}_{2}$ particies. Comparing Fig. 6(a) with $6(\mathrm{~b})$, we can see that the coercivity will be increased if the crystal anisotropy is taken into account. This means that the anisotropy forces due to combined effect of shape and crystal anisotropy are larger than those due to shape anisotropy.

According to Fig. 4, the reversal of magnetic moment is a parallel rotation mode when $\psi>\psi_{0}$. But in Fig. 6(a) we can find that in some $\psi<\psi_{0}$ angles the coercivity of the parallel rotation mode is equal to that of the fanning mode. For example at $n=2$, the coercivity of the parallel rotation mode is equal to the coercivity of the fanning mode at $13^{\circ} \leqslant \psi<32.5^{\circ}=\psi_{0}$. This is because the critical angle $\theta_{c}$ of the parallel rotation mode is just equal to the $\theta_{0}$ angle of the fanning mode in this $\psi$ range. That is, the applied field $H$ needed for parallel rotation reversal at $\theta>\theta_{0}$ is lower than that at $\theta_{6}$ in this $\psi$ range. For this reason, to $\mathrm{CrO}_{2}$ particles, the hysteresis loops of parallel rotation mechanism at various angles $\psi$ are the same as those of the fanning mechanism (Fig. 5) except for small $\psi\left(\psi<13^{\circ}\right.$ for $n=2, \psi<15^{\circ}$ for $n=3, \psi<25^{\circ}$ for $n=\infty$, etc. $)$.

\section{3. $\mathrm{Fe}_{3} \mathrm{O}_{4}$ and $\gamma / \mathrm{Fe}_{2} \mathrm{O}_{3}$ particles}

To elongated $\mathrm{Fe}_{3} \mathrm{O}_{4}$ and $\gamma-\mathrm{Fe}_{2} \mathrm{O}_{3}$ particles, from Eqs. (1), (13), (14), and (26), we have the relation between $H$ and $\theta$ :

$H \sin \theta=-\pi M_{s} K_{n}\left(\sin \theta \cos \theta \sin ^{2} \psi \cos ^{2} \phi+\cos 2 \theta \sin \psi \cos \psi \cos \phi-\sin \theta \cos \theta \cos ^{2} \psi\right)+\frac{1}{3} \pi M_{s} L_{n}(\cos 2 \phi-1)$

$$
\times \cos \theta \sin \theta+\frac{K_{1}}{M_{s}}\left(\frac{\partial A}{\partial \theta}\right)_{\phi} .
$$

And the solution of Eq. (27) is

$$
\sin \phi=0, \phi=0, \pi,
$$

or

$\frac{1}{4} \pi M_{s} K_{n}\left(4 \cos \phi \sin ^{2} \psi \sin ^{2} \theta+\sin 2 \theta \sin 2 \psi\right)-\frac{2}{3} \pi M_{s} L_{n} \sin ^{2} \theta \cos \phi+\left(K_{1} / M_{s}\right)\left[-\sin ^{4} \theta \sin ^{4} \psi \cos ^{3} \phi-3 \sin ^{3} \theta \cos \theta\right.$

$$
\begin{aligned}
& \times \sin ^{3} \psi \cos \psi \cos ^{2} \phi-3 \sin ^{2} \theta \cos ^{2} \theta \sin ^{2} \psi \cos ^{2} \psi \cos \phi-\sin \theta \cos ^{3} \theta \sin \psi \cos ^{3} \psi+2 \sin ^{4} \theta \sin ^{2} \psi \\
& \left.\times\left(\cos ^{3} \phi-\cos \phi \sin ^{2} \phi\right)+2 \sin ^{3} \theta \cos \theta \sin \psi \cos \psi\left(2 \cos ^{2} \phi-\sin ^{2} \phi\right)+2 \cos ^{4} \theta \sin ^{2} \theta \cos ^{2} \psi \cos \phi\right]=0 .
\end{aligned}
$$

Equation (33) indicates the parallel rotation mechanism and Eq. (34) is the faming mechanism. From Eqs. (1), (13), (14), and (31) the $\partial$ ranges for both mechanisms during the reversal of elongated $\mathrm{Fe}_{3} \mathrm{O}_{4}$ particles at various $\psi$ are shown in Fig. 7 . In this case, the upper bond angle $\psi_{0}$ decreases as $n$ is increased. This is opposite to that of $\mathrm{CrO}_{2}$. For $n=2, n=3$, and $n=\infty \mathrm{Fe}_{3} \mathrm{O}_{4}$ particles, the $\psi_{0}$ angles are $78^{\circ}, 66^{\circ}$, and $57^{\circ}$, respectively. The numerical calculations for the hysteresis loops of the faming mechanism at various angles $\psi$ of $n=2$ and $n=\infty \mathrm{Fe}_{3} \mathrm{O}_{4}$ particles are shown in Fig. 8. The hysteresis loops of elongated $\gamma-\mathrm{Fe}_{2} \mathrm{O}_{3}$ particles are similar to those of $\mathrm{Fe}_{3} \mathrm{O}_{4}$ particles. 
(a)

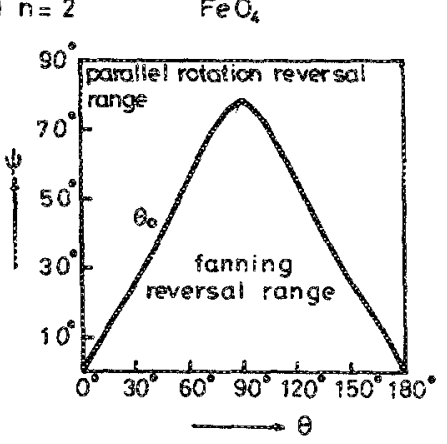

(b) $n=3$

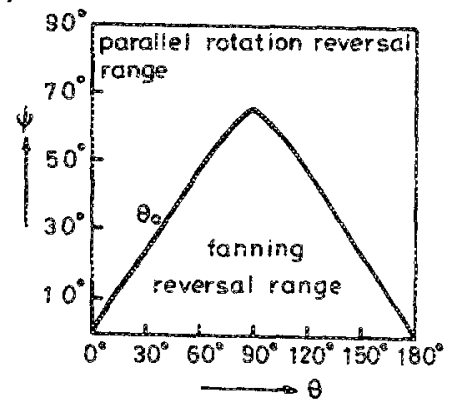

(c) $n=\infty$

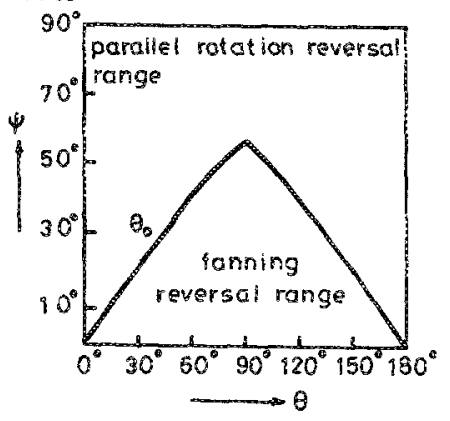

To the parallel rotation mechanism, the whole reversal process is described by $\phi=0$ and $\phi=\pi$ for any $\psi$. The representative hysteresis loops for $n=2$ and $n=\infty \mathrm{Fe}_{3} \mathrm{O}_{3}$ particles are shown in Fig. 9. Figure 10(a) shows the variation of coercivities with angle $\psi$ of $\mathrm{Fe}_{3} \mathrm{O}_{4}$ particles. Solid and dotted curves are calculated from the paraliel rotation mechanism and the fanning mechanism, respectively. Figure $10(\mathrm{~b})$ shows the shape-controlled coercivities of elongated $\mathrm{Fe}_{3} \mathrm{O}_{4}$ particles. Comparing Fig. 10(a) with 10(b) we can see that for the parallel rotation mechanism the coercivity will be increased if the crystal anisotropy is taken into account. However, for the fanning mechanism the coercivity is reduced when the crystal anisotropy is considered. This is because when the magnetizations of $\mathrm{Fe}_{3} \mathrm{O}_{4}$ particles are reversed by the parallel rotation mechanism, the anisotropy forces due to the combined effect of shape and crystal anisotropies are larger than those due to shape anisotropy. But when the rnagnetizations are reversed by the fanning mechanism, the results are opposite. Another difference between Figs. $10(a)$ and $10(b)$ is the transformation angle $\theta_{0}$. When $\psi>0$, the transformation angle of Fig. $10(a)$ is larger than that of Fig. 10(b) for any $n$. This is because the fanning reversal range becomes smaller when the crystal anisotropy is taken into account. In the case of elongated $\gamma-\mathrm{Fe}_{2} \mathrm{O}_{3}$ particles, the results are the same, as shown in Fig. 11. This is also opposite to that of $\mathrm{CrO}_{2}$ (see Fig. 6).

When crystal anisotropy is taken into account, the coercivities of $\mathrm{CrO}_{2}$ particles are increased both for parallel rotation mechanism and fanning mechanism. To $\mathrm{Fe}_{3} \mathrm{O}_{4}$ and $\gamma$ $\mathrm{Fe}_{2} \mathrm{O}_{3}$ particles, the coercivity of parallel rotation mechanism is increased but the coercivity of fanning mechanism is decreased.

The angular dependent coercivity curves of $\mathrm{CrO}_{2}$ and $\gamma$ $\mathrm{Fe}_{2} \mathrm{O}_{3}$ particies calculated from the fanning mechanism are in agreement with the experimental results of Corradi et al. ${ }^{24}$ for very diluted particle assemblies.

\section{Y. COERCIVTY OF RANDOMLY ORIENTEO PARTICLES}

We now examine the case of randomly oriented particles. To a randomly oriented chains assembly, the hysteresis loop can be produced by averaging the angle $\psi$. The average resolved value of the relative magnetization of randomly oriented chains for a given field $H$ will be
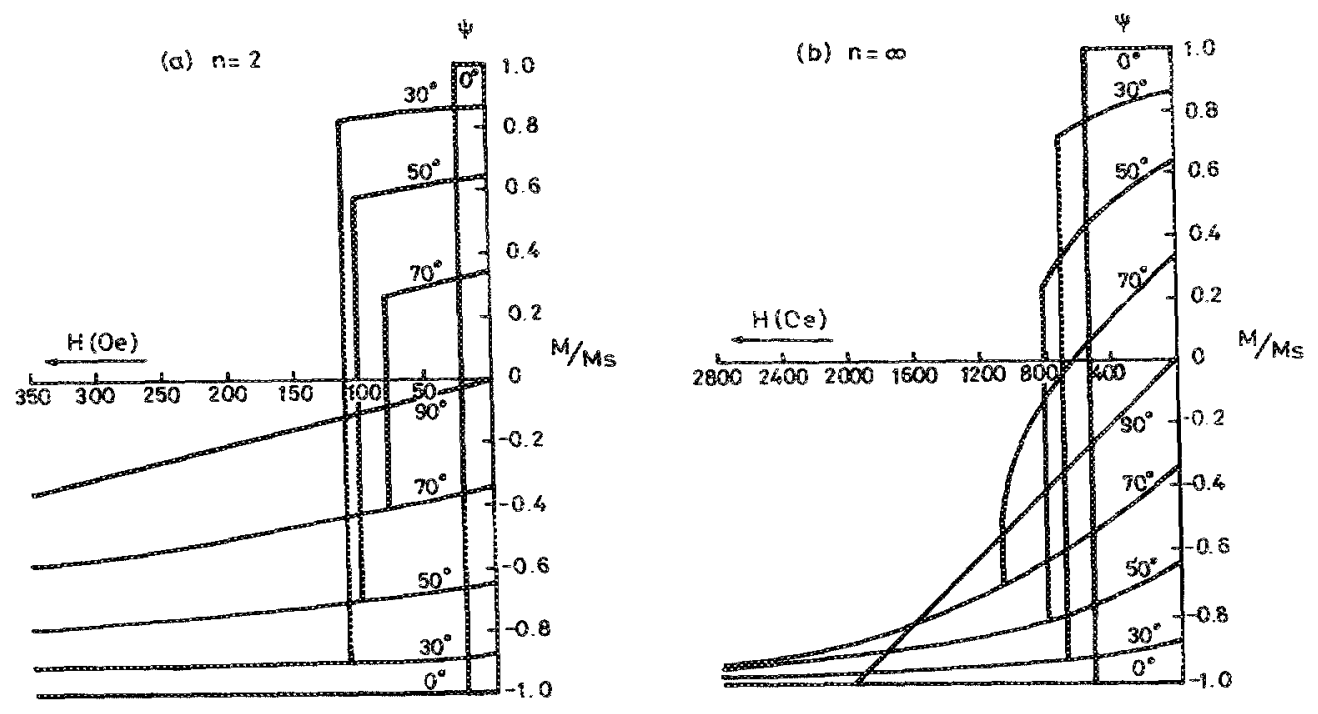

FIG. 8. Hysteresis loops of the fanning mechanism of $\mathrm{Fe}_{3} \mathrm{O}_{4}$ particle which oriented at various angles of $\psi$ to the applied field. Particle axial ratio: (a) $n=2$; (b) $n=\infty$. 

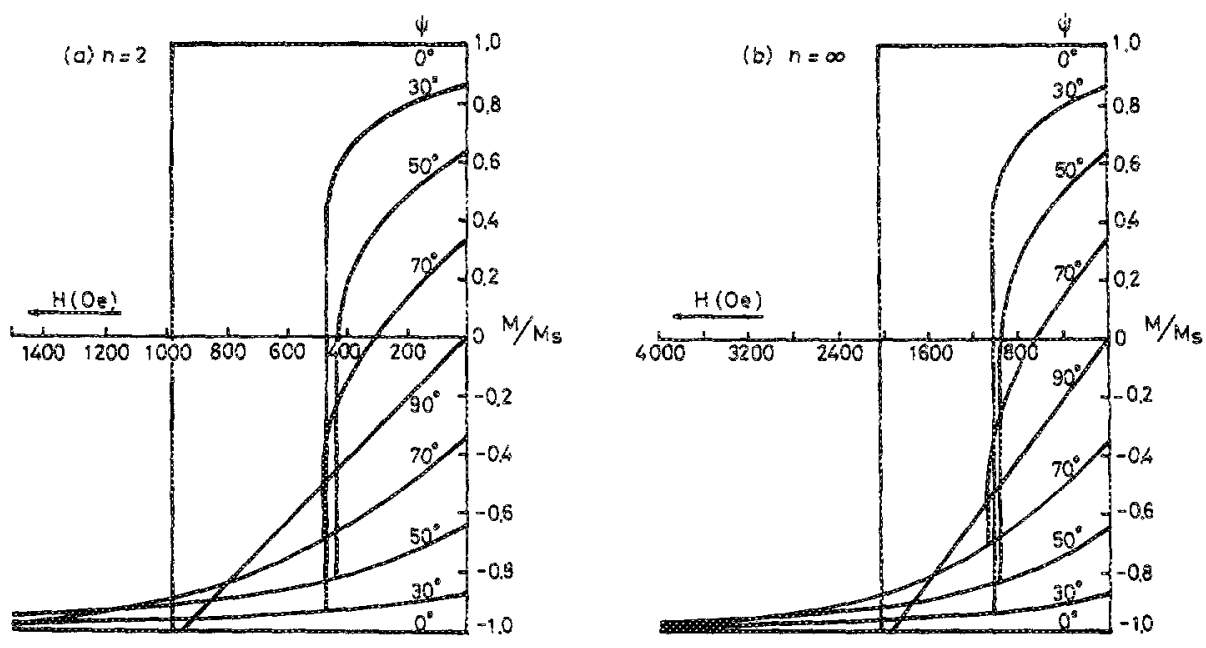

FIG. 9. Hysteresis loops of chain-of-spheres parallel rotation mechanism of $\mathrm{Fe}_{3} \mathrm{O}_{4}$ parkicle which oriented at various angle $\psi$ to the applied field. Particle axial ratio: (a) $n=2$; (b) $n=\infty$.

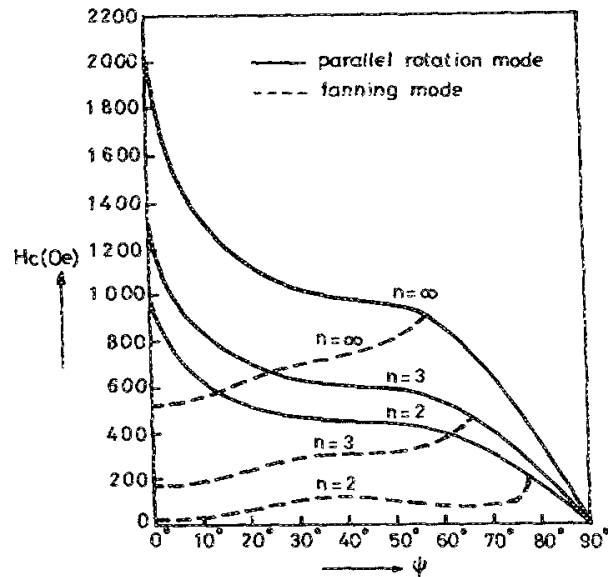

(a)

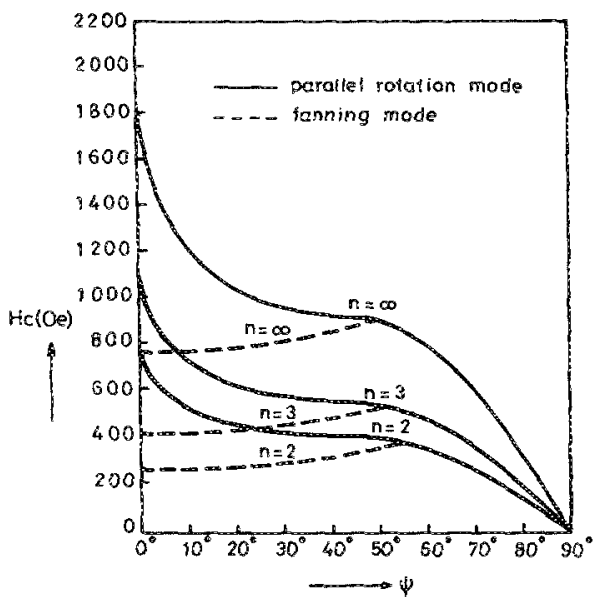

(b)

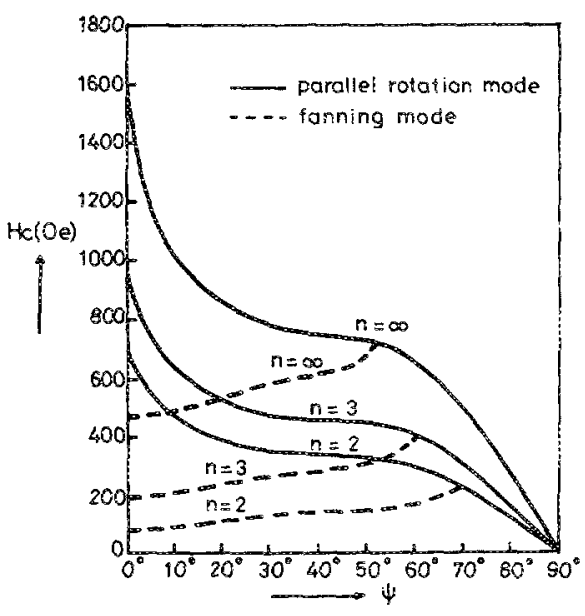

(A)

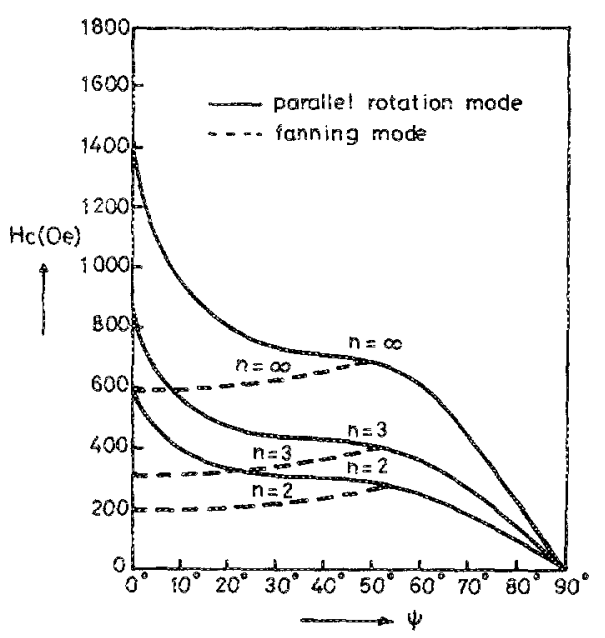

(B)
FIG. 10. Angular variation of the coercivity for $\mathrm{Fe}_{3} \mathrm{O}_{4}$ particles with various axial ratio. (a) Curves with mixed anisotropies of shape and crystal. (b) Curves with shape anisotropy.
FIG. 11. Angular variation of the coercivity for $\gamma-\mathrm{Fe}_{2} \mathrm{O}_{3}$ particles with various axial ratio. (a) Curves with mixed anisotropies of shape and crystal. (b) Curves with shape anisotropy. 


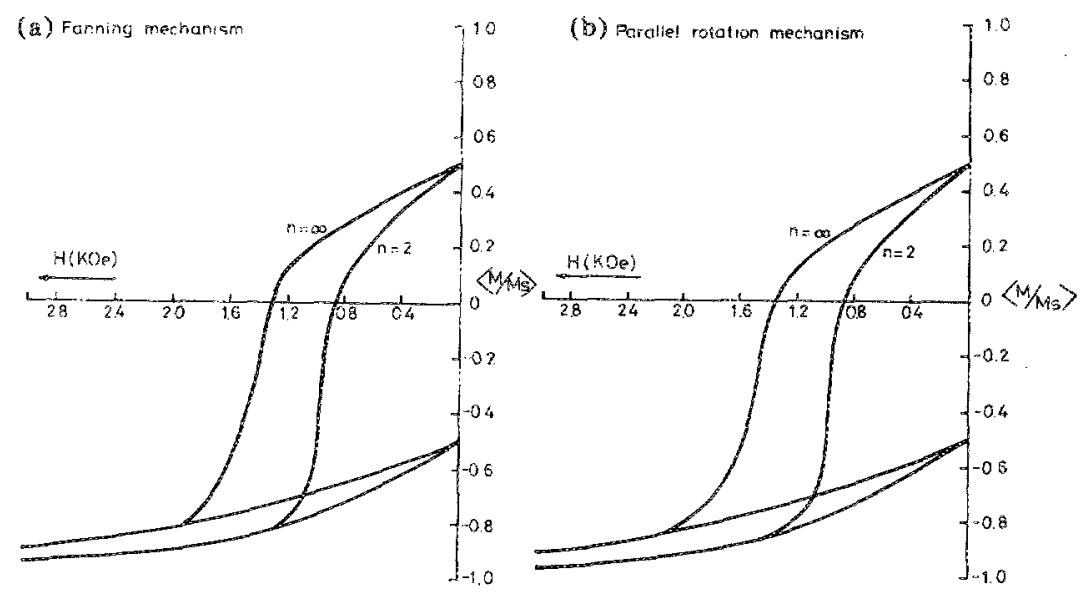

FEG. 12. Hysteresis loops of randomly oriented $\mathrm{CrO}_{2}$ particies calchiated from mixed effects of crystal and shape anisotropies. (a) Fanning mechanism. (b) Parallel rotation mechanism.

TABLE 1. Variation of relative magnetization with reverse ficld for rancomly oriented $n=2, n=5, n=10$, and $n=\infty$ CrO, particles.

(a) Fanning mechanism with mixed anisotropies.

\begin{tabular}{|c|c|c|c|c|c|c|c|c|}
\hline \multirow[t]{2}{*}{$H(\mathrm{Oe})$} & \multicolumn{4}{|c|}{$\begin{array}{c}\text { Upper branch } \\
(M / M)\end{array}$} & \multicolumn{4}{|c|}{$\begin{array}{c}\text { Lower branchi } \\
\left\langle M / M_{\checkmark}\right\rangle\end{array}$} \\
\hline & $n=2$ & $n=5$ & $n=: 10$ & $n=\infty$ & $n=2$ & $n \div 5$ & $n=10$ & $n=\infty$ \\
\hline 0 & +0.500 & +0.500 & +0.500 & +0.500 & -0.500 & -0.500 & -0.500 & -0.500 \\
\hline 200 & +0.422 & +0.441 & +0.446 & +0.451 & -0.563 & -0.547 & -0.543 & -0.539 \\
\hline 400 & +0.333 & +0.379 & +0.390 & +0.400 & -0.622 & $\ldots .594$ & -0.586 & -0.579 \\
\hline 600 & +0.226 & +0.308 & +0.327 & +0.344 & -0.675 & -0.636 & -0.626 & -0.616 \\
\hline 800 & +0.082 & +0.225 & +0.256 & +0.281 & -0.722 & -0.675 & -0.662 & -0.650 \\
\hline 900 & -0.078 & +0.178 & +0.215 & +0.246 & $\ldots .744$ & -0.693 & -0.680 & -0.667 \\
\hline 1000 & -0.571 & +0.123 & +0.171 & +0.209 & -0.764 & -0.711 & -0.696 & -0.682 \\
\hline 1100 & -0.732 & +0.057 & +0.120 & +0.167 & -0.784 & -0.728 & -0.712 & -0.697 \\
\hline 1200 & -0.801 & -0.080 & +0.060 & +0.121 & $\ldots 0.801$ & -0.744 & -0.727 & -0.712 \\
\hline 1300 & -0.818 & -0.491 & -0.124 & -0.001 & -0.818 & -0.760 & -0.742 & -0.726 \\
\hline 1400 & -0.834 & -0.713 & -0.478 & -0.227 & -0.834 & -0.774 & -0.756 & -0.740 \\
\hline 1500 & -0.849 & -0.784 & -0.655 & $\ldots-0.449$ & -0.849 & -0.788 & -0.770 & $\cdots 0.753$ \\
\hline 1600 & -0.862 & -0.802 & -0.744 & -0.573 & -0.862 & -0.802 & -0.783 & -0.765 \\
\hline 1900 & -0.894 & -0.838 & -0.889 & -0.805 & -0.894 & -0.838 & -0.819 & -0.805 \\
\hline 2200 & -0.914 & -0.869 & -0.850 & -0.831 & 0.914 & -0.869 & -0.850 & -0.831 \\
\hline 2900 & -0.939 & -0.913 & -0.901 & -0.888 & -0.939 & -0.913 & -0.901 & -0.838 \\
\hline 3400 & -0.948 & -0.929 & -0.921 & -0.911 & -0.948 & -0.929 & -0.921 & -0.911 \\
\hline 3900 & -0.953 & -0.940 & $\ldots 0.933$ & -0.926 & -0.953 & -0.940 & -0.933 & -0.926 \\
\hline 4400 & -0.957 & -0.946 & -0.941 & -0.936 & -0.957 & -0.946 & -0.941 & -0.936 \\
\hline
\end{tabular}

(b) Parallel rotation mechanism with mixed anisotropies

\begin{tabular}{|c|c|c|c|c|c|c|c|c|}
\hline \multirow[t]{2}{*}{$H(\mathrm{Oe})$} & \multicolumn{4}{|c|}{$\begin{array}{c}\text { Upper branch } \\
\langle M / M,\rangle\end{array}$} & \multicolumn{4}{|c|}{$\begin{array}{c}\text { Lower branch } \\
\left\langle M / M_{v}\right\rangle\end{array}$} \\
\hline & $n:=2$ & $n=5$ & $n=10$ & $n=\infty$ & $n=2$ & $n=5$ & $n=10$ & $n=\infty$ \\
\hline 0 & +0.500 & +0.500 & +0.500 & +0.500 & -0.500 & -0.500 & -0.500 & -0.500 \\
\hline 200 & +0.416 & +0.436 & +0.442 & +0.446 & -0.564 & -0.547 & $\ldots 0.543$ & -0.539 \\
\hline 400 & +0.324 & +0.371 & +0.383 & +0.393 & -0.626 & -0.596 & -0.588 & -0.581 \\
\hline 600 & +0.213 & +0.298 & +0.318 & +0.335 & -0.682 & -0.641 & -0.630 & -0.619 \\
\hline 800 & +0.066 & +0.213 & +0.244 & +0.270 & -0.732 & -0.682 & -0.668 & -0.656 \\
\hline 900 & -0.059 & +0.164 & +0.202 & +0.234 & -0.755 & -0.702 & -0.687 & -0.673 \\
\hline 1000 & -0.542 & +0.108 & +0.157 & +0.196 & -0.777 & $\ldots .721$ & -0.704 & -0.690 \\
\hline 1100 & $\cdots 0.738$ & +0.041 & +0.105 & +0.153 & -0.798 & -0.738 & -0.721 & -0.706 \\
\hline 1200 & -0.784 & $\ldots 0.062$ & +0.044 & +0.106 & -0.817 & -0.756 & -0.738 & $-0.72 k$ \\
\hline 1300 & -0.836 & -0.527 & -0.041 & +0.050 & -0.836 & -0.772 & $\ldots 0.754$ & -0.736 \\
\hline 1400 & $\ldots .853$ & -0.683 & $\ldots 0.255$ & $\ldots 0.093$ & -0.853 & 0.788 & -0.769 & -0.751 \\
\hline 1500 & $\ldots 0.869$ & -0.748 & -0.567 & -0.346 & -0.869 & -0.803 & -0.783 & -0.765 \\
\hline 1600 & -0.884 & -0.785 & -0.737 & -0.545 & -0.884 & -0.818 & -0.797 & $\ldots 0.778$ \\
\hline 1900 & -0.918 & -0.857 & $-\ldots .036$ & -0.779 & -0.918 & -0.857 & -0.836 & $\ldots 0.816$ \\
\hline 2200 & -0.939 & -0.891 & -0.870 & -0.850 & -0.939 & $\ldots-0.891$ & -0.870 & -0.850 \\
\hline 2700 & -0.959 & -0.928 & -0.915 & -0.897 & $\ldots 0.959$ & . 0.928 & -0.915 & -0.897 \\
\hline 3200 & -0.970 & -0.948 & -0.938 & -0.927 & -0.970 & -0.948 & -0.938 & -0.927 \\
\hline 3700 & -0.976 & $-0.96 i$ & -0.954 & -0.945 & -0.976 & -0.961 & -0.954 & -0.945 \\
\hline 4200 & -0.981 & -0.969 & -.0 .964 & -0.957 & -0.981 & -0.969 & -0.964 & -0.957 \\
\hline 4700 & -0.984 & $\cdots 0.975$ & -0.971 & -0.965 & -0.984 & -0.975 & -0.971 & -0.965 \\
\hline
\end{tabular}


$\left\langle\left(M / M_{s}\right)_{H}\right\rangle=\left\langle(\cos \theta)_{H}\right\rangle=\int_{0}^{\pi / 2}(\cos \theta)_{H} \sin \psi d \psi$

For a given $\psi$, the value of $(\cos \theta)_{H}$ can be obtained from the graphical hysteresis loop.

\section{A. $\mathrm{CrO}_{2}$ particles}

According to the numerical calculations, the hysteresis loops of randomly oriented $n=2$ and $n=\infty \mathrm{CrO}_{2}$ particles for the fanning mechanism and the parallel rotation mechanism are shown in Figs. 12(a) and 12(b), respectively.
These loops are plotted by using the data calculated for loops of constant $\psi$ in $5^{\circ}$ intervals. Table $I$ presents data from which these curves are produced. Since the $\psi$ range of the fanning reversal mode is small to compare with the whole $\alpha$ range, as shown in Fig. 6(a), the curves of Figs. 12 (b) and $12(3)$ are similar.

The coercivity of randomly oriented $n=10 \mathrm{CrO}_{2}$ particles at zero packing fraction $H_{c}(0)$ observed by Umeki et $a .^{25}$ is 605 Oe. The coercivities of the parallel rotation mechanism and the fanning mechanism calculated from mixed anisotropies are 1260 and 12450 e, respectively. They are all higher than the experimental value of Umeki et al.

TABL.E II. Variation of relative magnetization with reverse field for randomiy oriented $n=2, n=5, n=10, n=\infty$ Fe, $\mathrm{O}_{4}$ and $\gamma_{2}$ Fe $\mathrm{O}_{3}$ particles $($ parallel rotation mechanism with mixed anisotropies).

(a) $\mathrm{Fe}_{3} \mathrm{O}_{4}$ particles

\begin{tabular}{|c|c|c|c|c|c|c|c|c|}
\hline \multirow[t]{2}{*}{$H(\mathrm{Oe})$} & \multicolumn{4}{|c|}{$\begin{array}{c}\text { Upper branch } \\
\left\langle M / M_{s}\right\rangle\end{array}$} & \multicolumn{4}{|c|}{$\begin{array}{c}\text { Lower branch } \\
\left\langle M / M_{s}\right\rangle\end{array}$} \\
\hline & $n=2$ & $n=5$ & $n=10$ & $n=\infty$ & $n=2$ & $n=5$ & $n=10$ & $n=\infty$ \\
\hline 0 & +0.500 & +0.500 & +0.500 & +0.500 & -0.500 & -0.500 & -0.500 & -0.500 \\
\hline 100 & +0.424 & +0.451 & +0.457 & +0.461 & -0.566 & -0.550 & -0.546 & -0.541 \\
\hline 200 & +0.340 & +0.404 & +0.416 & +0.426 & -0.621 & -0.584 & -0.575 & -0.568 \\
\hline 300 & +0.239 & +0.352 & +0.372 & +0.388 & -0.662 & -0.615 & -0.603 & -0.593 \\
\hline 400 & +0.096 & +0.294 & +0.324 & +0.347 & -0.701 & -0.643 & -0.629 & -0.617 \\
\hline 500 & -0.560 & +0.227 & +0.270 & +0.302 & -0.737 & -0.671 & -0.654 & -0.640 \\
\hline 600 & -0.774 & +0.147 & +0.210 & +0.254 & -0.774 & -0.696 & -0.678 & -0.662 \\
\hline 700 & -0.804 & +0.038 & +0.138 & +0.198 & -0.804 & $-0.72 !$ & -0.701 & -0.683 \\
\hline 800 & -0.835 & -0.516 & +0.043 & +0.134 & -0.835 & -0.745 & -0.722 & -0.703 \\
\hline 900 & -0.866 & -0.734 & -0.504 & +0.053 & -0.866 & -0.767 & -0.743 & -0.722 \\
\hline 1050 & -0.901 & -0.789 & $-0.7 \AA 7$ & -0.308 & -0.901 & -0.789 & -0.763 & -0.741 \\
\hline 1300 & -0.951 & -0.853 & -0.839 & -0.776 & -0.951 & -0.853 & -0.819 & -0.793 \\
\hline 1600 & -0.972 & -0.906 & -0.870 & $-0.84 !$ & -0.972 & -0.906 & -0.870 & -0.841 \\
\hline 1900 & -0.978 & -0.937 & -0.911 & -0.885 & -0.978 & -0.937 & -0.911 & -0.885 \\
\hline 2500 & -0.986 & -0.966 & -0.958 & -0.939 & --0.986 & -0.966 & -0.958 & -0.939 \\
\hline 3100 & -0.989 & -0.977 & -0.970 & -0.962 & -0.989 & -0.977 & -0.970 & -0.962 \\
\hline 3700 & -0.991 & -0.983 & -0.979 & -0.973 & -0.991 & -0.983 & -0.979 & -0.973 \\
\hline
\end{tabular}

(b) $\mathrm{\gamma}-\mathrm{Fe}_{2} \mathrm{O}_{3}$ particles

\begin{tabular}{|c|c|c|c|c|c|c|c|c|}
\hline \multirow[t]{2}{*}{$H(O e)$} & \multicolumn{4}{|c|}{$\begin{array}{c}\text { Upper branch } \\
\left\langle M / M L_{s}\right\rangle\end{array}$} & \multicolumn{4}{|c|}{$\begin{array}{c}\text { Lower branch } \\
\left\langle M / M_{3}\right\rangle\end{array}$} \\
\hline & $n=2$ & $n=5$ & $n=10$ & $n=\infty$ & $n=2$ & $n=5$ & $n=10$ & $n=\infty$ \\
\hline 0 & +0.500 & +0.500 & $\div 0.500$ & +0.500 & -0.500 & -0.500 & -0.500 & -0.500 \\
\hline 50 & +0.448 & +0.466 & +0.470 & +0.473 & -0.557 & -0.536 & -0.532 & -0.528 \\
\hline 100 & $\div 0.394$ & +0.436 & +0.444 & +0.450 & -0.591 & -0.560 & -0.552 & -0.546 \\
\hline 150 & +0.335 & +0.404 & +0.416 & +0.426 & -0.624 & -0.582 & -0.572 & -0.564 \\
\hline 200 & +0.268 & +0.369 & $\div 0.387$ & +0.402 & -0.654 & -0.604 & -0.592 & -0.582 \\
\hline 250 & +0.187 & +0.333 & +0.357 & +0.376 & -0.683 & -0.624 & -0.610 & -0.599 \\
\hline 300 & +0.081 & +0.293 & +0.324 & +0.348 & -0.710 & -0.644 & -0.628 & -0.615 \\
\hline 350 & -0.422 & +0.250 & +0.290 & +0.319 & -0.736 & -0.663 & -0.646 & -0.631 \\
\hline 400 & -0.730 & +0.203 & +0.252 & +0.289 & -0.761 & -0.681 & -0.663 & -0.646 \\
\hline 450 & -0.778 & +0.148 & +0.212 & +0.256 & -0.785 & -0.699 & -0.679 & -0.661 \\
\hline 500 & -0.807 & +0.084 & +0.166 & +0.221 & -0.807 & -0.716 & -0.695 & -0.676 \\
\hline 600 & -0.851 & -0.511 & +0.052 & +0.141 & -0.851 & -0.749 & -0.725 & -0.704 \\
\hline 700 & -0.897 & -0.745 & -0.516 & +0.033 & -0.897 & -0.780 & -0.753 & -0.730 \\
\hline 800 & -0.924 & -0.809 & -0.739 & -0.516 & -0.924 & -0.809 & -0.780 & -0.755 \\
\hline 1000 & -0.956 & -0.862 & -0.829 & -0.779 & -0.956 & -0.862 & -0.829 & -0.801 \\
\hline 1300 & -0.974 & -0.923 & -0.898 & -0.863 & -0.974 & -0.923 & -0.898 & -0.863 \\
\hline 1600 & -0.982 & -0.952 & -0.934 & -0.913 & -0.982 & -0.952 & -0.934 & -0.913 \\
\hline 2200 & -0.988 & -0.974 & -0.966 & -0.956 & -0.988 & -0.974 & -0.966 & -0.956 \\
\hline 2800 & -0.991 & -0.982 & -0.978 & -0.972 & $-0.99 ?$ & -0.982 & -0.978 & -0.972 \\
\hline
\end{tabular}




\section{8. $\mathrm{Fe}_{3} \mathrm{O}_{4}$ and $\gamma-\mathrm{Fe}_{2} \mathrm{O}_{3}$ particles}

Tables $I(a)$ and $U(b)$ are the data of the parallel rotation mechanism for randomly oriented $\mathrm{Fe}_{3} \mathrm{O}_{4}$ and $\gamma$ - $\mathrm{Fe}_{2} \mathrm{O}_{3}$ particles where $n=2,5,10$, and $s$. The corresponding hysteresis loops of $n=2$ and $n=\infty$ are shown in Fig. 13. Tables III (a) and III(b) are the data of fanning mechanism for randomly oriented $\mathrm{Fe}_{3} \mathrm{O}_{4}$ and $\gamma-\mathrm{Fe}_{2} \mathrm{O}_{3}$ particles with various axial ratios. The corresponding hysteresis loops of $n=2$ and $n=\infty$ are shown in Fig. 14.

The coercivity of very dilated $n=6$ randomly oriented
$\mathrm{Fe}_{3} \mathrm{O}_{4}$ particles observed by Davis ${ }^{26}$ is about 450 Oe. To $\mathrm{Fe}_{3} \mathrm{O}_{4}$, the coercivities of randomily oriented $n=6$ particles calculated from mixed anisotropries for the parallel rotation mechanism and the fanning mechanism are 740 and $525 \mathrm{Oe}$, respectively. It is clear that the value of the fanning mechanism is closer to Davis' experimental coercivity.

The coercivities of very diluted $n=5$ randomly oriented $\gamma-\mathrm{Fe}_{2} \mathrm{O}_{3}$ particles observed by Bottoni et al. ${ }^{27}$ are about $310 \mathrm{Oe}$, and the coercivity $H_{c}(0)$ of $n=10 \gamma \cdot \mathrm{Fe}_{2} \mathrm{O}_{3}$ particles investigated by Umeki et al. ${ }^{25}$ is 500 oe. The calculated coercivities of mixed anisotropies for fanning mechanism of

TABLE IIL. Variation of relative magnetization with reverse feld for randomly oriented $n=2, n=5, n=10, n=\infty F_{1} \mathrm{O}_{4}$ and $\gamma^{-F e} \mathrm{O}_{3}$ particles (fanning mechanism with nixed anisotropies).

(a) $\mathrm{Fe}_{3} \mathrm{O}_{4}$ particies

\begin{tabular}{|c|c|c|c|c|c|c|c|c|}
\hline \multirow[t]{2}{*}{$H(\mathrm{Oe})$} & \multicolumn{4}{|c|}{$\begin{array}{c}\text { Upper branch } \\
\left\langle M M_{s}\right\rangle\end{array}$} & \multicolumn{4}{|c|}{$\begin{array}{c}\text { Lower branch } \\
\left(M / M_{1}\right)\end{array}$} \\
\hline & $n=2$ & $n=5$ & $n=10$ & $n==\infty$ & $n=2$ & $n=5$ & $n=10$ & $n=\infty$ \\
\hline 0 & +0.500 & +0.500 & +0.500 & -0.500 & --0.500 & -0.500 & -0.500 & -0.500 \\
\hline 50 & +0.421 & +0.474 & +0.476 & +0.478 & -0.526 & -0.514 & -0.512 & -0.510 \\
\hline 100 & +0.161 & +0.451 & +0.457 & +0.461 & -0.558 & -0.539 & -0.530 & -0.526 \\
\hline 150 & -0.285 & +0.428 & +0.437 & +0.444 & -0.587 & -0.554 & -0.547 & -0.541 \\
\hline 200 & -0.508 & +0.404 & +0.416 & +0.426 & $\ldots 0.616$ & -0.573 & -0.564 & -0.556 \\
\hline 250 & -0.612 & +0.378 & +0.394 & +0.407 & -0.644 & -0.591 & -0.580 & -0.570 \\
\hline 300 & -0.670 & +0.350 & +0.372 & +0.388 & -0.670 & -0.609 & -0.596 & -0.585 \\
\hline 350 & -0.696 & +0.316 & +0.348 & +0.368 & -0.696 & -0.626 & -0.611 & -0.599 \\
\hline 400 & -0.720 & +0.254 & +0.324 & +0.347 & --0.720 & -0.643 & -0.626 & -0.612 \\
\hline 450 & -0.744 & +0.162 & +0.279 & +0.325 & -0.744 & -0.660 & -0.641 & -0.625 \\
\hline 500 & -0.767 & +0.041 & +0.231 & +0.302 & -0.767 & -0.676 & -0.655 & -0.638 \\
\hline 600 & -0.812 & -0.428 & +0.046 & +0.214 & -0.812 & -0.766 & -0.683 & -0.664 \\
\hline 700 & -0.855 & -0.635 & -0.303 & +0.073 & -0.855 & -0.736 & -0.710 & -0.688 \\
\hline 800 & -0.891 & -0.731 & -0.634 & -0.142 & -0.891 & -0.753 & -0.735 & -0.711 \\
\hline 1000 & -0.931 & -0.815 & -0.782 & -0.670 & -0.931 & -0.815 & -0.782 & -0.755 \\
\hline 1300 & -0.959 & -0.883 & -0.846 & -0.814 & -0.959 & -0.883 & --0.846 & -0.814 \\
\hline 1600 & -0.972 & -0.923 & -0.899 & -0.865 & -0.972 & -0.923 & -0.899 & -0.865 \\
\hline 2200 & -0.983 & -0.959 & $\ldots 0.946$ & -0.929 & -0.983 & -0.959 & -0.946 & -0.929 \\
\hline 2800 & -0.988 & -0.974 & -0.966 & -0.956 & -0.988 & -0.974 & -0.966 & -0.956 \\
\hline
\end{tabular}

(b) $\gamma$ - $\mathrm{Fe}_{2} \mathrm{O}_{3}$ particles

\begin{tabular}{|c|c|c|c|c|c|c|c|c|}
\hline \multirow[t]{2}{*}{$H(\mathrm{Oe})$} & \multicolumn{4}{|c|}{$\begin{array}{l}\text { Upper branch } \\
\left\langle M / M_{\mathrm{s}}\right\rangle\end{array}$} & \multicolumn{4}{|c|}{$\begin{array}{c}\text { Lower branch } \\
\left\langle M / M_{*}\right\rangle\end{array}$} \\
\hline & $n=2$ & $n=5$ & $n:=10$ & $n=\infty$ & $n=2$ & $n=5$ & $n==10$ & $n==\infty$ \\
\hline 0 & +0.500 & +0.500 & +0.500 & +0.500 & -0.500 & -0.500 & -0.500 & -0.500 \\
\hline 50 & +0.448 & +0.466 & +0.470 & +0.473 & -0.538 & -0.521 & -0.518 & -0.515 \\
\hline 100 & +0.355 & +0.436 & +0.444 & +0.450 & $\ldots 0.580$ & -0.548 & -0.541 & -0.535 \\
\hline 150 & +0.147 & +0.404 & +0.416 & +0.426 & -0.619 & -0.573 & -0.563 & -0.555 \\
\hline 200 & -0.520 & +0.369 & +0.387 & +0.402 & -0.657 & -0.597 & -0.585 & -0.575 \\
\hline 250 & -0.628 & +0.333 & +0.357 & +0.376 & -0.692 & -0.621 & -0.606 & -0.593 \\
\hline 3000 & -0.702 & +0.293 & +0.324 & +0.348 & -0.725 & -0.643 & -0.626 & -0.611 \\
\hline 350 & -0.749 & +0.211 & +0.290 & +0.319 & -0.757 & -0.665 & -0.645 & -0.629 \\
\hline 400 & -0.787 & +0.017 & +0.243 & +0.289 & -0.787 & -0.686 & -0.664 & -0.646 \\
\hline 450 & -0.815 & -0.308 & +0.172 & +0.256 & -0.815 & -0.706 & -0.682 & -0.662 \\
\hline 500 & -0.843 & -0.470 & -0.017 & +0.214 & -0.843 & -0.726 & -0.700 & -0.678 \\
\hline 600 & -0.892 & -0.687 & -0.490 & -0.042 & -0.892 & -0.763 & -0.734 & -0.709 \\
\hline 700 & -0.920 & -0.783 & -0.692 & -0.501 & -0.920 & -0.797 & -0.765 & -0.738 \\
\hline 800 & -0.939 & -0.829 & -0.777 & -0.691 & -0.939 & -0.829 & -0.795 & -0.766 \\
\hline 1000 & -0.961 & -0.885 & -0.848 & -0.816 & -0.961 & -0.885 & -0.848 & -0.816 \\
\hline 1300 & -0.976 & -0.932 & -0.910 & -0.880 & -0.976 & -0.932 & -0.910 & -0.880 \\
\hline 1600 & -0.983 & -0.955 & -0.940 & -0.922 & -0.983 & -0.955 & -0.940 & -0.922 \\
\hline 2200 & -0.989 & -0.975 & -0.968 & -0.958 & -0.989 & -0.975 & -0.968 & -0.958 \\
\hline 2800 & -0.992 & -0.983 & -0.979 & -0.973 & -0.902 & -0.983 & -0.979 & -0.973 \\
\hline
\end{tabular}




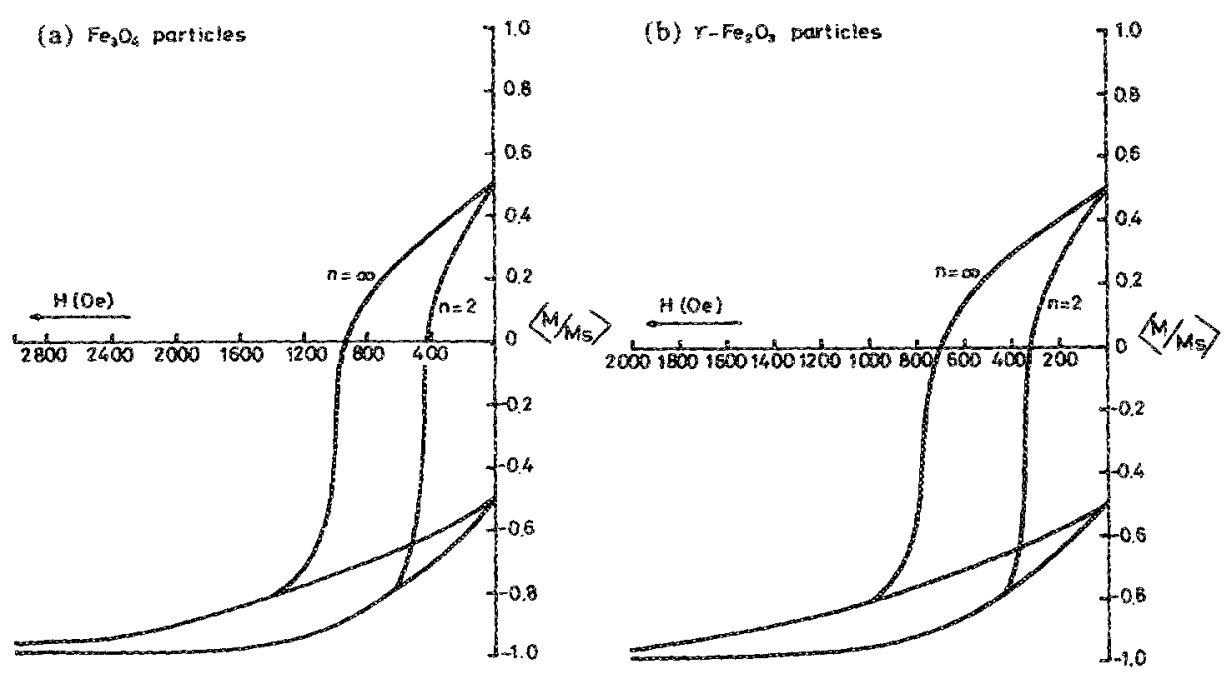

FIG. 13. Hysteresis loops of parallel rotation mechanism for randomly oriented $\mathrm{Fe}_{3} \mathrm{O}_{4}$ and $\gamma-\mathrm{Fe}_{2} \mathrm{O}_{3}$ particles caiculated from mixed effects of crystal and shape anisotropies. (a) $\mathrm{Fe}_{3} \mathrm{O}_{4}$ particles. (b) $\gamma-\mathrm{Fe}_{2} \mathrm{O}_{3}$ particles.

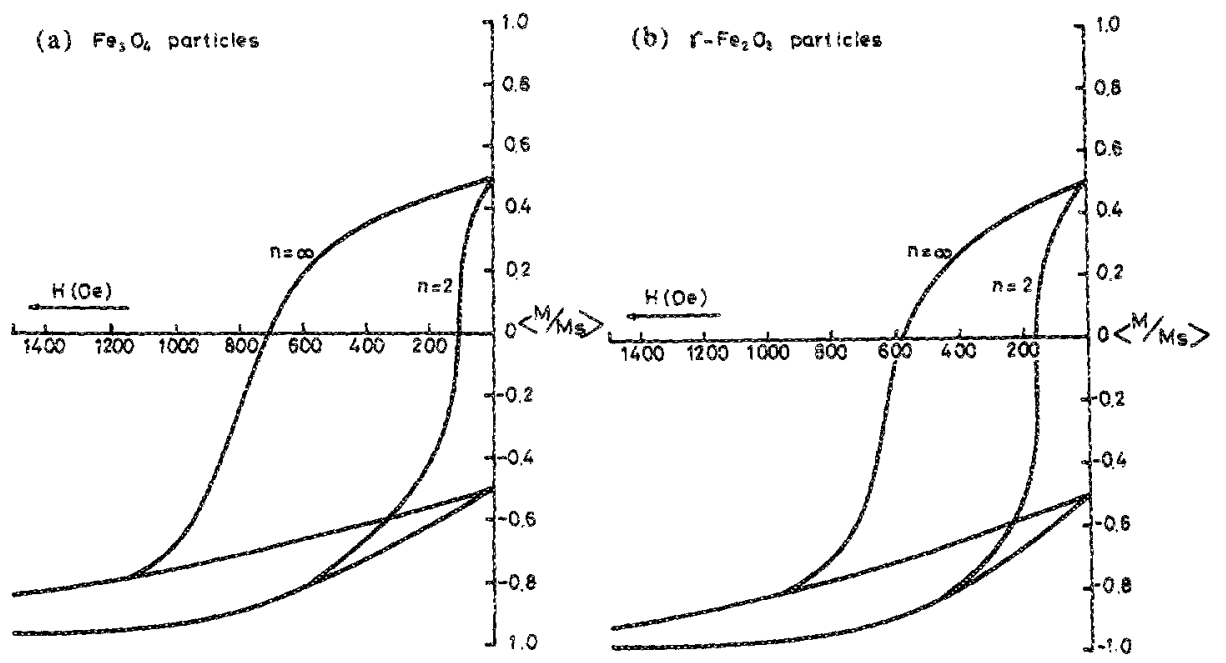

FIG. 14. Hysteresis loops of the fanning mechanism for randomly oriented $\mathrm{Fe}_{3} \mathrm{O}_{4}$ and $\gamma-\mathrm{Fe}_{2} \mathrm{O}_{3}$ particles calculated from mixed effects of crystal and shape anisotropies. (a) $\mathrm{Fe}_{3} \mathrm{O}_{4}$ particles. (b) $\gamma-\mathrm{Fe}_{2} \mathrm{O}_{3}$ particles.

$n=5$ and $n=10 \gamma-\mathrm{Fe}_{2} \mathrm{O}_{3}$ particles are 410 and $490 \mathrm{Oe}$, respectively. The agreement between theoretical values and experimental results is good. However, from chain-ofspheres parallei rotation mechanism with mixed anisotropies, the calculated coercivities of $n=5$ and $n=10 \gamma-\mathrm{Fe}_{2} \mathrm{O}_{3}$ particles are 720 and 8200 , respectively. They are all larger than the experimental values of Bottoni et al. and Umeki et al.

\section{CONCLUSION}

In this paper, the chain-of-spheres model is modified by taking crystal anisotropy into account. The case of easy crystal axis at some angle to the particle axis is studied. The coercivities of aligned and randomly oriented particles due to mixed anisotropies of shape and crystal are calculated. During magnetization reversal, the rotation-of-sphere moments shall overcome the energy barriers that are produced by the mixed anisotropies. Coercivity of the chain is equivalent to the applied feld that just overcome these energy barriers.

To $\mathrm{CrO}_{2}$ particles, the coercivity calculated from mixed anisotropies is larger than that calculated from shape anisotropy. To $\mathrm{Fe}_{3} \mathrm{O}_{4}$ and $\gamma-\mathrm{Fe}_{2} \mathrm{O}_{3}$ particles, the coercivity calculated from mixed anisotropies for fanning mechanism is smaller than that calculated from shape anisotropy, but the result is opposite for parallel rotation mechanism. For $\mathrm{CrO}_{2}$ particles, the contribution of crystal anisotropy to their coercivity is large, but this contribution is small for $\mathrm{Fe}_{3} \mathrm{O}_{3}$ and $\gamma-\mathrm{Fe}_{2} \mathrm{O}_{3}$ particles.

It has been shown that the coercivities of $\mathrm{CrO}_{2}, \mathrm{Fe}_{3} \mathrm{O}_{4}$, and $\gamma-\mathrm{Fe}_{2} \mathrm{O}_{3}$ particles calculated from the fanning mechanism reasonably agree with the published experimental values. However, the coercivities calculated from the chain-ofspheres parallel rotation mechanism are usually larger than the published measured values. 
1E. C. Stoner and E. P. Wohlfarth, Philos. Trans. R. Soc. London 240, 599 (1948).

${ }^{2}$ E. H. Frei, S. Shtrikman, and D. Treves, phys, Rev. 106. 446 (1957).

${ }^{3}$ I. S. Jacobs and C. P. Bean, Phys. Rev, 100, 1060 (1955)

D. F. Eagle and J. C. Mallinson, J. Appl. Phys. 38, 995 (1967).

${ }^{5}$ F. E. Luborsky, E. F. Fullam, and D. S. Hallgren, J. Appl. Phys. 29, 989 (1958).

${ }^{6} \mathrm{~L}$. Néel, Compt. Rend 224, 1488 (1947).

F. E. Luborsky, L. I. Mendelsohn, and T. O. Paine, J. Appl. Phys. 28, 344 (1957).

'F. E. Luborsky, I. Appl. Phys. 32, i71S (1961).

75. J. Darnell, j. Appl. Phys. 32, 1269 (1961).

${ }^{10}$ I. S. Jacobs and F. E. Luborsky, J. Appl. Phys. 28, 467 (1957).

"P. C. Kuo and C. Y. Chang, J. Appl. Fhys. 57,4678 (1985); ibid. 59, 995 (1986).

${ }^{12}$ K. A. Wihelmi and O. Jonsson, Acta. Chem. Scand. 12, 1532 (1958).

${ }^{13}$ E. Köster, IRE Conf. Proc. 26, 213 (1973).

${ }^{14}$ D. S. Rodball, J. Phys. Soc. Jpn. 21, 1244 (1966).

${ }^{15}$ D. S. Rodbell, R. C. De Vries, W. D. Barber, and R. W. De Blois, I. Appl.
Phys. 38, 4542 (1967)

${ }^{16} \mathrm{G}$. Bate, in Magnetic Oxides, edited by D. I. Craik (Wiley, London, 1975). p. 689.

${ }^{17}$ T. K. Mevat, F. A. Fox, and A. J. F. Boyle, I. Appl. Phys 39, 5703 (1968).

${ }^{18 \mathrm{H}}$. Takei and S. Chiba, J. Phys. Soc. Jpn. 21, 1255 (1966).

19J. Hurt, A. Amendola, and R. E. Smith, J. Appl, Phys. 37, 1170 (1966).

${ }^{20} \mathrm{G}$. W. Oosterhout, Acta. Cryst. 13, 932 (1960).

21. Smit and H. P.J. Wijn, Ferrites (Wiley, New Vork, 1959), p. 163.

22J. E. Knowles, Proc. ICE 3 (Japan), 627 (1980).

${ }^{23}$ J. E. Knowles, IEEE Trans. Magn. MAG-16, 62 (1980).

${ }^{24}$ A. R. Corradi, S. T. Andress, J. R. French, G. Bottoni, D. Candolfo, A. Cecchetri, and F. Masol, IEEE Trans. Magn. MA.20, 33 (1984).

${ }^{25} \mathrm{~S}$. Umeki, H. Sugihara, Y. Yaketomi, and Y. Imaoka, TEEE Trans. Magn. MAE $=17,3014(1981)$

76P. M. Davis, J. Appl. Phys. 51, 594 (1980).

${ }^{27}$ G. Bottoni, D. Candolfo, A. Cecchett, and F. Masoh, IEEE Trans. Magn. MAG 8,770 (1972). 